\title{
Towards Integration of Heterogeneous Controllers in an IOT-based Automation System
}

\author{
Handy Wicaksono ${ }^{1, *}$, Petrus Santoso ${ }^{1}$, Iwan Handoyo Putro ${ }^{1}$, Ivan Surya Hutomo ${ }^{2}$, \\ and Pricilia Alvina ${ }^{1}$ \\ ${ }^{1}$ Petra Christian University, Electrical Engineering Department, Jl. Siwalankerto 121-131 \\ Surabaya 60236, Indonesia \\ ${ }^{2}$ Electrical Engineering and Computer Science Department, National Chiao Tung University, \\ 1001 University Road, Hsinchu,Taiwan 300, ROC
}

\begin{abstract}
Heterogeneous controllers (such as: various brands of PLCs and microcontrollers), are existed in an automated system. This research propose a framework to integrate them by using NodeRed, an open source project to gather various devices and services in an IOT setting. The research framework was applied successfully in three cases: a smart home prototype for elderly, a smart door using face recognition method, and an automated system for multiple houses in separated locations.
\end{abstract}

Keywords: Communication, data, internet of things, smart home, programmable logic controller

\section{Introduction}

Usage of heterogeneous controllers are unavoidable in a complex environment (e.g. a smart house, a manufacturing factory) because there is no "silver bullet" to control everything in such an environment. Consider a smart house dedicated for the elderly which may have these various controllers: a Programmable Logic Controller (PLC) to control its lighting and heating system, a dedicated controller to control a mobile robot which patrols through every rooms, and a controller to recognize the voice of an elderly person.

In the past, especially in factory automation field, it is difficult to integrate those controllers as most automation vendors prefer closed and proprietary system so their customers keep coming back to them and use their products only. However, this "closed ecosystem" becomes less popular because customers want to choose any product which has the best quality and the cheapest price altogether [1]. Additionally, the Fourth Industrial Revolution (FIR) movement also encourages Internet of Things (IOT). This means that all vendors should provide a way, so their devices can be accessed by the other various devices. This research propose a framework that is able to facilitate communication between multivendor devices.

A modern automated system must be able to store its data from the plant floor into a database. This is not the case in the past, where data acquisition and visualization are enough. The stored data is useful for analytical, tracking, or evaluation purpose. It is also

\footnotetext{
*Corresponding author: handy@petra.ac.id
} 
can be fed to a machine learning algorithm to get meaningful insight that is useful for prediction (e.g. predictive maintenance for devices in a factory [2]). Currently, this research only provide a mechanism to collect data from heterogenous devices, store them in a database system, and visualize them to help system operators. In the future, data will be analysed via machine learning.

The research framework was applied on three cases. Firstly, a prototype of a smart home for elderly is controlled by different brand of PLCs in an IOT ecosystem. It is also equipped by a mobile robot which is connected to the IOT. Secondly, a smart door prototype which is able to recognise the face of the homeowner (or his friends and families). Lastly, an automated system to control several buildings in separated locations. A simple Human Machine Interface (HMI) is developed. It is also able to display data in trend and record them into a simple database. To achieve smooth integration of heterogeneous controllers and store their data into a database, a Node-Red based solution were chosen. Node-Red is an open source project initiated by IBM which aims to connect various hardware devices, APIs and online services. Figure 1 shows the first NodRed-based system applied in the first case.

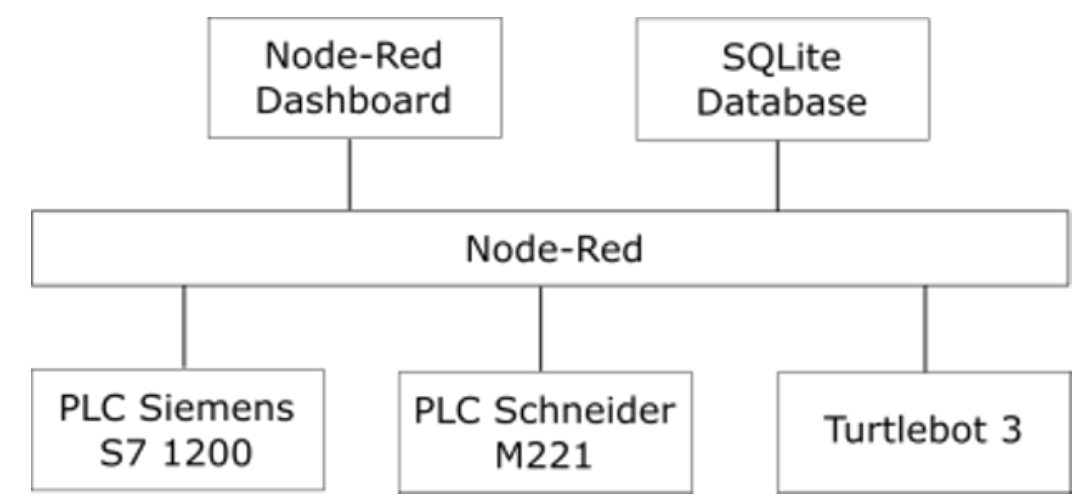

Fig. 1. NodeRed-based framework to integrate various hardware devices, a dashboard, and a database.

\section{Literature review}

Instead of living in a nursing home, many elderly want to stay in their own houses, so researchers want to assist them with various smart home technology [3]. Majumder et al. [4] proposed a smart home for elderly which is equipped with a home gateway which connects to: various home appliances (for user convenience and security), wearable sensors embedded on elderly bodies, dan data collection system (for analysis and learning purpose). This research want to combine this home automation system with a mobile robot customized for elderly needs.

Security is an important aspect of a smart building. Recently, a face recognition approach is getting more attention and considered to be closer to human intelligence than other approach [5]. Lim, Rotinsulu and Santoso [6] perform face recognition to access a room, however it does not connected to any physical door. It also still uses a laptop and a webcam, so it is not portable to be relocated to other locations. Haar cascade and local binary pattern histogram algorithm can be used to detect faces.

Currently, many people own more than one houses, which needs to be monitored closely for security purpose. Sirait [7] develops a system based on Raspberry Pi to monitor the building security, however it is only for one building. While Santoso [8] proposed a system to monitor two S7 1200 PLCs and a PC with Wonderware InTouch. Despite 
successful result, his configuration is relatively expensive so it might not affordable with a person who has a tight budget.

\section{A smart home prototype for elderly}

This research propose a smart home prototype for an elderly which consists of an automated home customized for elderly and a mobile robot (as a mobile communication medium to medical staffs or families).

\subsection{Design and implementation}

A NodeRed has been used in this system. It is originally developed to wire IOT subsystems, however, currently many open projects that provide nodes of many non-IOT controllers such as PLCs. Once all devices connected through Node-Red, their data can be visualized via a Node-Red dashboard and stored into a database system. Figure 1 shows the diagram of Node-Red based system which consists of PLC Siemens S7 1200, PLC Schneider M221, a Raspberry Pi which controls a Turtlebot 3 (a mobile robot in Robot Operating System environment), a Node-Red dashboard, and an SQLite database.

\subsection{Initial experiment results}

The experiment begins by connecting a S7 1200 Siemens PLC to Node-Red installed in Simatic IOT 2040 (an IOT gateway from Siemens). The IP configuration of PC, IOT 2040, and S7 1200 is shown in Figure 2a, while Figure 2b shows the simple experimental setup. The S7 communication has been used as the protocol to connect S7 1200 to Node-Red. Figure $2 \mathrm{c}$ contains the flow where a node (S7 Read) reads the discrete input (\%I1.1), a constant and an analog input (\%IW64). The result is displayed on both simple dashboard (see Figure 2d) and debugging screen. It is also stored into a simple file. Similar setup are also used to conduct experiment to connect an M221 Schneider PLC to Node-Red using Modbus TCP protocol. The simple experiments show that it is possible to integrate two PLCs from various vendors and visualize their data in a simple form.

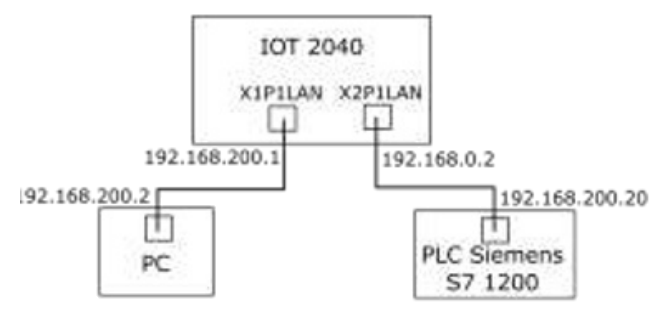

(a)

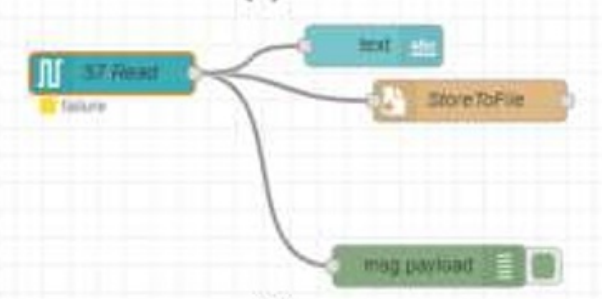

(c)

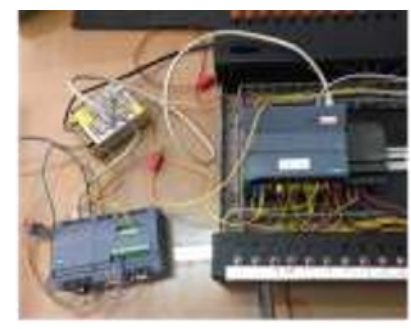

(b)

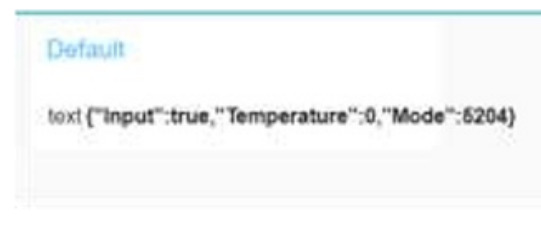

(d)

Fig. 2. Experimental configuration and initial result in connecting S7 1200 to Node-Red. 


\section{A smart door protoype with face recognition capability}

This research develop a smart door prototype which is able to recognise a person. Then the recognised person can control the door using his voice. Design, implementation, and experiment results will be describe in the next subsection.

\subsection{Design and implementation}

The prototype have four subsystems, whose block diagram is shown on Figure 3. The first one is the smart door subsystem, consists of a motor servo, a smart lock mechanism, and a door chassis. It is controlled by a RaspberryPi 3. The face recognition subsystem is the second one. It is based on Haar Cascade and Local Binary Pattern Histogram algorithms implemented using OpenCV library in a Raspberry Pi3. It utilizes a Raspberry Camera V2 as its camera. The third subsystem is a speech recognition system, implemented using a Google AIY Voice Kit. The recognition is done by mechanisms in Google Cloud, not in local controller. The last one is a notification subsystem, which utilises Google Assistant, Firebase and Blynk to send notifications to owner's mobile phone. As in the first case, NodeRed is functioned to gather all (hardware) devices and (software) services in this second case.

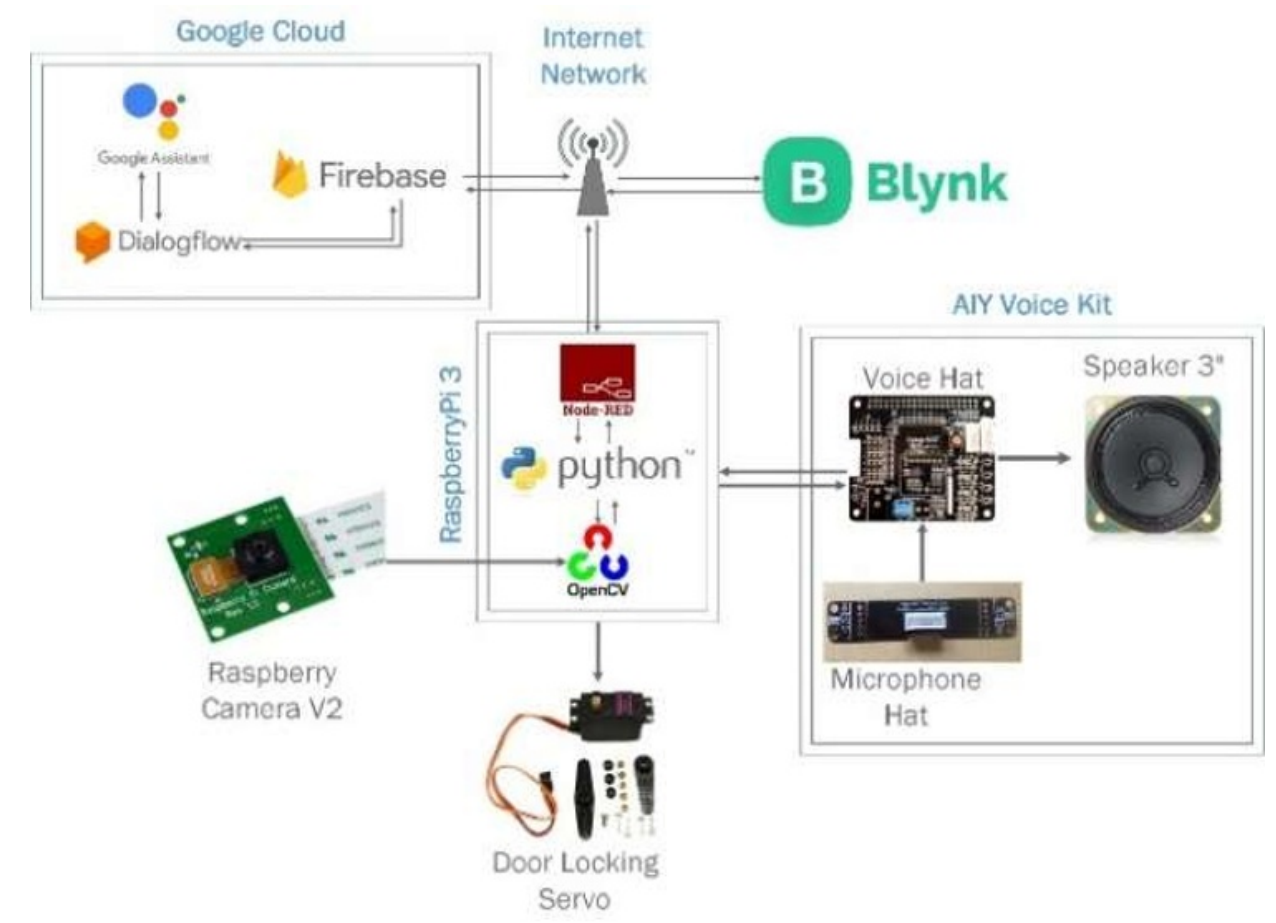

Fig. 3. Block diagram of smart door system [9].

\subsection{Experiment results}

The main focus in this research is the face recognition to open the smart door. The existing algorithms were used in OpenCV library: Haar cascade and Local Binary Pattern Histogram, however this research experiment with several parameters in the algorithms 
(MinNeighbour, Scale Factor) and setting configuration (the camera distance to a person) to get the best result. Figure 4 shows the recognition result of a known person (average confidence: $70 \%$ ) and unknown person (average confidence: $30 \%$ ).
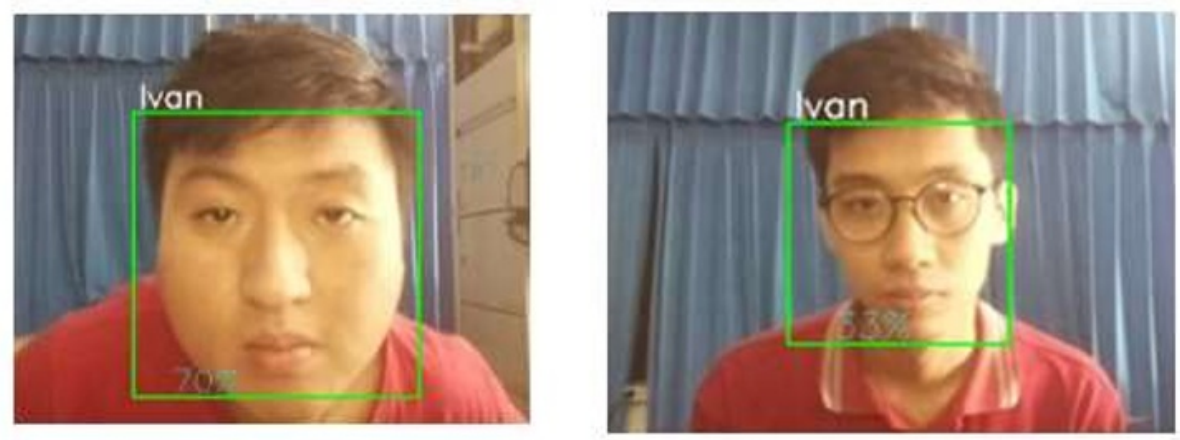

Fig. 4. Face recognition result of a known and unknown person. [9].

\section{Automation of multiple houses in separated locations}

Another application of the research framework is automation of multiple houses in separated locations. Design, implementation and results will be describe below.

\subsection{Design and implementation}

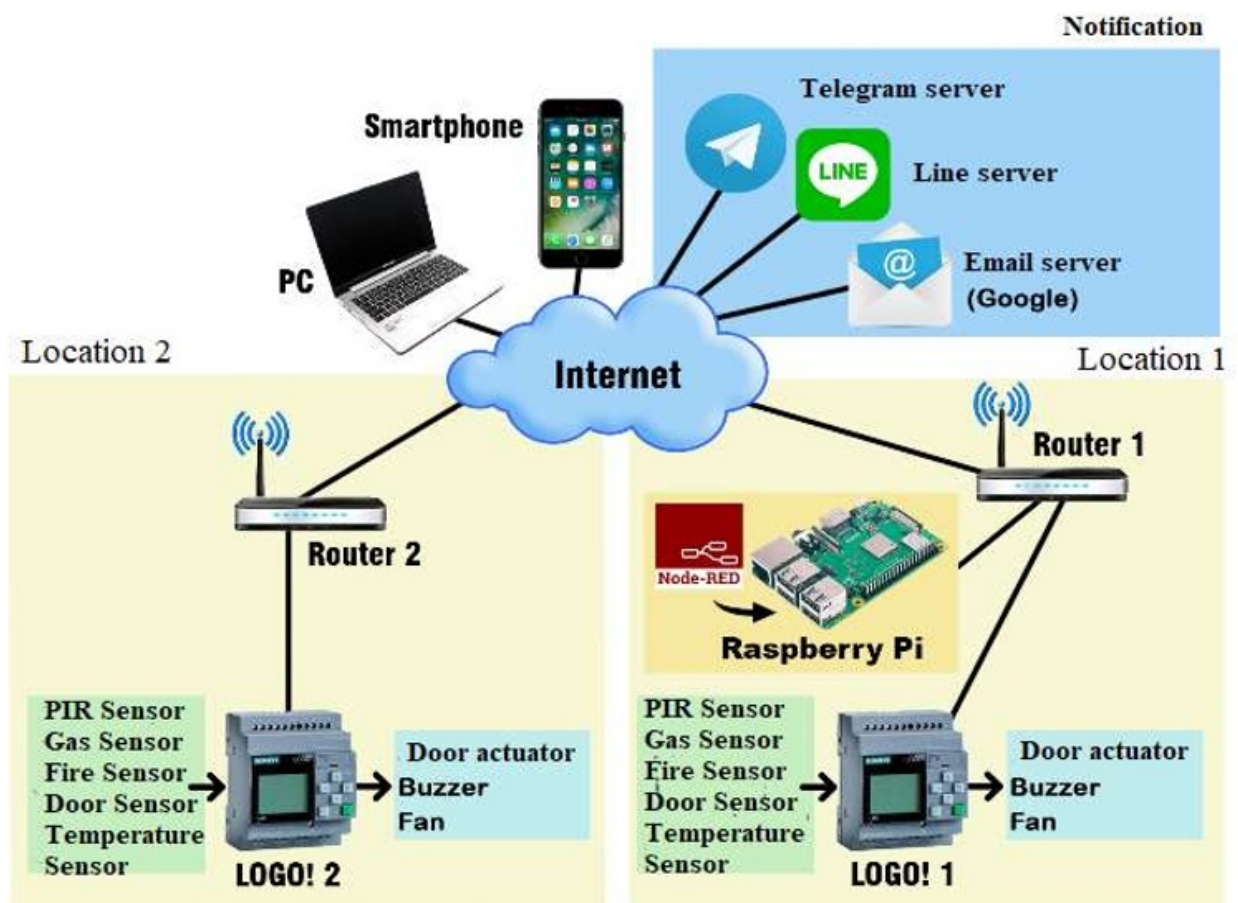

Fig. 5. Block diagram of automation of multiple houses in separated locations [10]. 
The protoype consists of two houses, each of them is controlled by a LOGO! Controller. It is also equipped with various sensors (e.g. smoke sensor, gas sensor, activity detection sensor, door sensor) and actuators (e.g. fan). As in previous two cases, NodeRed (installed in a RaspberryPi) is used to collect data from LOGO! Controllers, display it as a trend and send it as notification in Telegram messages, LINE messages, and email (Google mail). The proposed block diagram is shown on Figure 5.

\subsection{Experiment results}

The main function of this system is to notify the building owner if there is an intruder trying to enter their property or if there is fire or gas leaking happens in the building. When those unwanted events happen, the owner receives notification in their email or instant message service (LINE, Telegram). The status of each sensor is displayed in a browser, and the alarms (abnormal events) are recorded in a simple database (see Figure 6 for the detail).

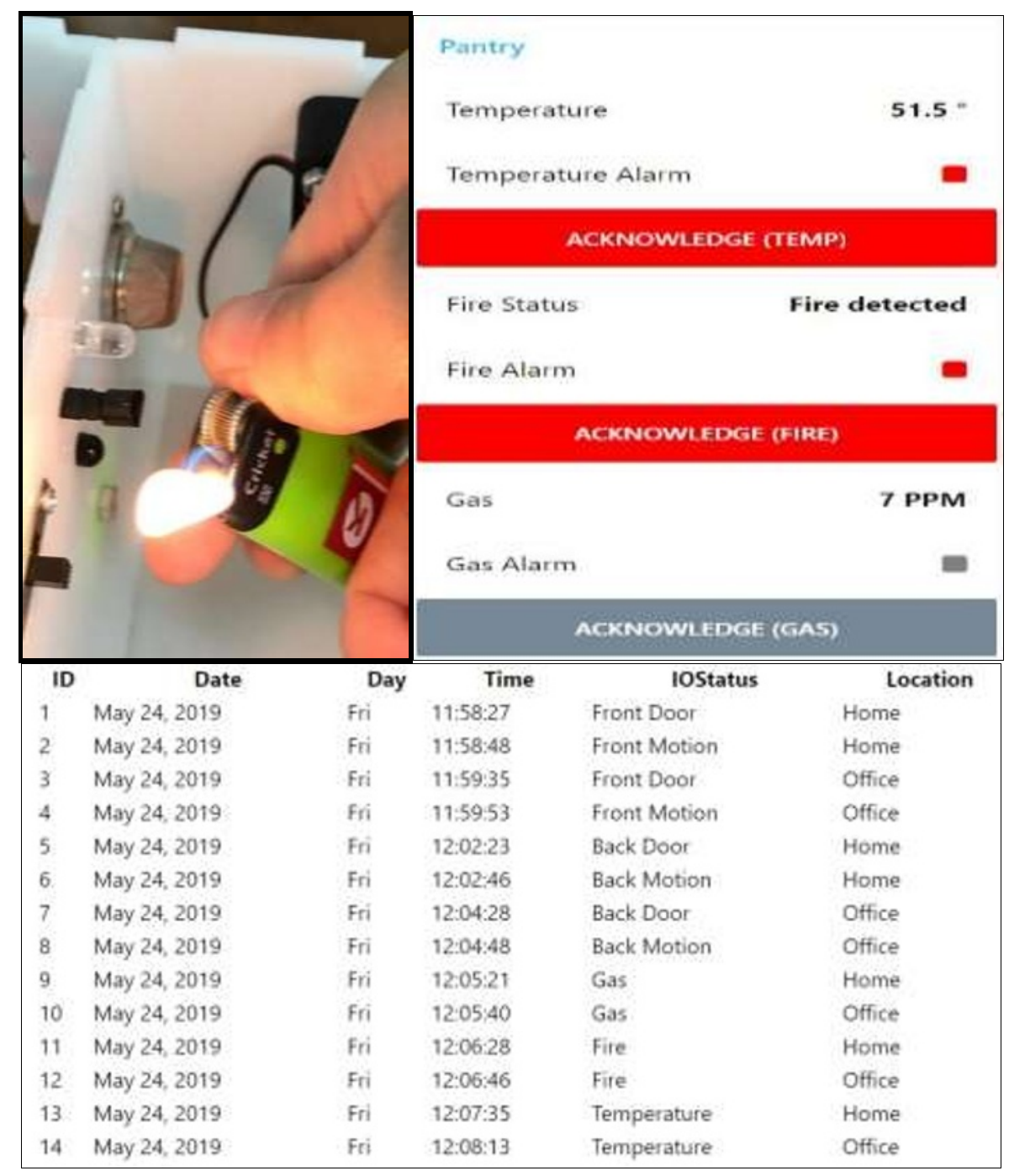

Fig.6. Status of a fire sensor in a browser and alarm records in a simple database [10]. 


\section{Conclusions}

A NodeRed-based framework was proposed to gather various (hardware) devices and (software) services in an IOT scenario. The research framework was applied in three cases: a smart home prototype for elderly, a smart door protoype using a face recognition method, and an automated system for multiple houses in separated locations. The heteregenous controllers (such as: S7 1200 PLC, M221 PLC, LOGO!, a Raspberry Pi microcontroller) are connected successfully in this research framework.

\section{References}

1. M. Noura, M. Atiquzzaman and M. Gaedke. Mobile Networks and Applications, 24,3:796-809(2019). https://link.springer.com/article/10.1007/s11036-018-1089-9

2. B. Kroll, D. Schaffranek, S. Schriegel, O. Niggemann. System modeling based on machine learning for anomaly detection and predictive maintenance in industrial plants. Proceedings of the 2014 IEEE Emerging Technology and Factory Automation (EFTA) (Barcelona, Spain, 2014). IEEE, 1-7(2014).

https://ieeexplore.ieee.org/document/7005202

3. J.V. Hoof, G. Demiris, E.J.M. Wouters. Handbook of smart homes, health care and well-being. Cham, Swiss: Springer International Publishing (2017). p. 674. https://link.springer.com/referencework/10.1007\%2F978-3-319-01904-8

4. S. Majumder, E. Aghayi, M. Noferesti, H. Memarzadeh-Tehran, T. Mondal, Z. Pang, et al. Sensors, 17,11:1-32(2017). https://www.mdpi.com/1424-8220/17/11/2496

5. M.P. Beham, S.M.M. Roomi. Int. J. Pattern Recognit. Artif. Intell., 27,04(2013). https://doi.org/10.1142/S0218001413560053

6. R. Lim, F. Rotinsulu, P. Santoso. Appl. Mech. Mater., 815:398-402(2015). https://www.scientific.net/AMM.815.398

7. F. Sirait. Jurnal Teknologi Elektro, Universitas Mercu Buana, 6,1:55-60(2015). [in Bahasa Indonesia]. http://publikasi.mercubuana.ac.id/index.php/jte/article/view/790

8. Y.N. Santoso. Sistem SCADA berbasis internet untuk model otomasi bangunan. [Undergraduate Thesis]. Teknik Elektro, Universitas Kristen Petra, Surabaya (2012). [in Bahasa Indonesia]. https://onesearch.id/Record/IOS4677.JATIM000000002307814

9. I.S. Hutomo. Integrasi smart door lock dengan face recognition berbasis RaspberryPi 3 dilengkapi fitur Google Assistant. [Integration of smart door lock with face recognition based on RaspberryPi 3 features Google Assistant] [Undergraduate Thesis]. Teknik Elektro, Universitas Kristen Petra, Surabaya (2019). [in Bahasa Indonesia]. https://dewey.petra.ac.id/catalog/digital

10. P. Alvina, H. Wicaksono, P. Santoso. SinarFe7, 2,1:376-381(2019). [in Bahasa Indonesia]. http://ejournal.fortei7.org/index.php/SinarFe7/article/view/75 